This article was published in Energy and Environmental Science, 7 (12), 4044-4052, 2014. http://dx.doi.org/10.1039/c4ee02160f

\title{
On the stability enhancement of cuprous oxide water splitting photocathodes by low temperature steam annealing
}

\author{
J. Azevedo, ${ }^{\text {ab }}$ L. Steier, ${ }^{c}$ P. Dias, ${ }^{b}$ M. Stefik, ${ }^{d}$ C. T. Sousa, ${ }^{b}$ J. P. Araújo, ${ }^{b}$ A. Mendes, ${ }^{a}$ \\ M. Graetzel' ${ }^{\mathrm{a}}$ and S. D. Tilley*c
}

aLEPABE - Faculdade de Engenharia, Universidade do Porto, Rua Dr Roberto Frias, 4200465 Porto, Portugal

bIFIMUP and IN-Institute of Nanoscience and Nanotechnology, Departamento de F'ısica e Astronomia, Universidade do Porto, Rua do Campo Alegre 687, 4169-007 Porto, Portugal

'Institut des Sciences et Ingénierie Chimiques, Ecole Polytechnique Fédérale de Lausanne (EPFL), CH-1015 Lausanne, Switzerland. E-mail: david.tilley@epfl.ch dDepartment of Chemistry and Biochemistry, University of South Carolina, Columbia, South Carolina, 29208, USA

Given the intermittent nature of solar radiation, the large-scale use of solar energy requires an efficient energy storage solution. So far, the only practical way to store such large amounts of energy is in the form of a chemical energy carrier, i.e., a fuel. Photoelectrochemical (PEC) cells offer the ability to convert solar energy directly into chemical energy in the form of hydrogen. Cuprous oxide $\left(\mathrm{Cu}_{2} \mathrm{O}\right)$ is being investigated for photoelectrochemical solar water splitting since it has a band gap of $2.0 \mathrm{eV}$ with favorable energy band positions for water cleavage; it is abundant and environmentally friendly. A major challenge with $\mathrm{Cu}_{2} \mathrm{O}$ is its limited chemical stability in aqueous environments. We present a simple and low-cost treatment to create a highly stable photocathode configuration for $\mathrm{H}_{2}$ production, consisting of steam treatment of the multilayer structures. The role of this treatment was investigated and the optimized electrodes have shown photocurrents over $-5 \mathrm{~mA} \mathrm{~cm} \mathrm{~cm}^{-2}$ with $90 \%$ stability over more than $50 \mathrm{~h}$ of light chopping (biased at $0 \mathrm{~V}_{\mathrm{RHE}}$ in $\mathrm{pH} 5$ electrolyte). 


\section{Introduction}

Due to the emerging necessity to find sustainable alternatives to fossil fuels, numerous investigations are being conducted in order to find efficient means to extract energy from the largest renewable energy source, the Sun. One of the biggest challenges with solar energy is that it is only available during daytime. Hence, the large-scale use of solar energy requires an efficient energy storage solution. So far, the only practical way to store such large amounts of energy is in the form of a chemical energy carrier, i.e., a fuel.

Photoelectrochemical (PEC) water splitting cells offer the ability to convert solar energy directly into chemical energy, stored in the chemical bonds of hydrogen and oxygen. Hydrogen is one of the prime candidates as a future energy carrier. ${ }^{1}$ It can be produced from renewable energy sources by (photo)electrolysis of water, stored and transported, and then used in a fuel cell to generate electricity on demand, yielding water without generating any harmful by-products. Alternatively, the renewable hydrogen can be reacted with carbon dioxide to generate carbonbased fuels. PEC water splitting has been much improved since the initial reports, ${ }^{2,3}$ and nowadays researchers aim at finding inexpensive, efficient and stable materials to perform PEC water splitting. ${ }^{4-10}$

Cuprous oxide, $\mathrm{Cu}_{2} \mathrm{O}$, is abundant, cheap and environmentally friendly and it absorbs a significant part of the solar spectrum with a $2 \mathrm{eV}$ bandgap, with favorable energy band positions for water cleavage. It has good p-type conductivity and it can be processed by low-cost methods such as electrodeposition, ${ }^{11}$ thus fulfilling all the necessary criteria for a promising photocathode, except for one - stability in aqueous environments.

Recently, we have presented a multilayer configuration, using $\mathrm{Cu}_{2} \mathrm{O}$ as the photoactive material, which addressed the instability problem of this material under working condi-tions. ${ }^{11,12}$ The samples were prepared on gold-coated F:SnO2 (FTO) glass substrates. The photoelectrode consisted of a photovoltaic $p-n$ junction of $p$-type Cu2O and n-type Al:ZnO (AZO) coated with a TiO2 protective layer, and activated for hydrogen evolution with electrodeposited $\mathrm{Pt}$ nanoparticles, producing photocurrents up to $-7.6 \mathrm{~mA} \mathrm{~cm}^{-2}$, under simulated solar AM 1.5 illumination when biased at $0 \mathrm{~V}_{\mathrm{RHE} .}{ }^{11}$ Despite the conformal protective layer of $\mathrm{TiO} 2$ deposited by atomic layer deposition (ALD), a decay of performance over the first 20 minutes was observed. No evidence of $\mathrm{Cu}_{2} \mathrm{O}$ degradation was observed and the photocurrent decline was attributed to electron traps in the protective $\mathrm{TiO} 2$ aggravated by its amorphous state. Although the stability was still far from ideal, this configuration has revealed a new strategy that inspired us to investigate improved overlayers for better stability.

In the first approach, higher deposition temperature of the TiO2 ALD deposited layer revealed stability improvements. ${ }^{12}$ In that work, deposition of the TiO2 layer at $150{ }^{\circ} \mathrm{C}$ enhanced the stability of the resulting photocathode when compared to the lower deposition temperature $\left(120^{\circ} \mathrm{C}\right)$. Furthermore, in a recent work on the detachment of platinum from the surface of the photocathode upon illumination, $\mathrm{RuO}_{\mathrm{x}}$ as a co-catalyst has been investigated. ${ }^{13} \mathrm{RuO}_{\mathrm{x}}$ is more resistant to heavy metal ion poisoning from impurities ${ }^{14}$ in the electrolyte and our porous electrodeposited film provides a high surface area that is crucial for efficient charge extraction from the TiO2 underlayer. ${ }^{13}$ This co-catalyst yielded a stable photocurrent of $-5 \mathrm{mAcm}^{-2}$ when 
biased at $0 \mathrm{~V}_{\mathrm{RHE}}$ during $8 \mathrm{~h}$ of light chopping. ${ }^{13}$ The improved catalyst/TiO2 interface allowed a record-breaking stability of $\mathrm{Cu}_{2} \mathrm{O}$-based photocathodes for water reduction.

From these previous reports, we developed an under-standing for the influence of $\mathrm{TiO} 2$ synthesis conditions on the photocathode stability. An alternative approach for further improving the photoelectrode stability targets improving the quality of the $\mathrm{TiO} 2$ protective layer with post-synthetic treatments. Heating the samples at high temperatures $\left(\approx 400{ }^{\circ} \mathrm{C}\right)$ could convert the amorphous $\mathrm{TiO} 2$ into a crystalline phase, which we expect would be more stable in the slightly acidic aqueous electrolyte. However, as the annealing temperature increases above $200{ }^{\circ} \mathrm{C}$, the performance of the photocathode is diminished, perhaps due to the loss of doping in the cuprous oxide, which is based on copper vacancies.

This work investigates an innovative low temperature steam treatment for improving the stability of $\mathrm{Cu}_{2} \mathrm{O}$-based photo-electrodes protected with largely amorphous TiO2 layers. Low temperature steam post-synthetic treatments were implemented, and have demonstrated greatly enhanced stabilities compared with non-treated samples. Photocurrents over $5 \mathrm{~mA} \mathrm{~cm}{ }^{-2}$ were achieved with only a $10 \%$ loss over its initial photocurrent value during more than $50 \mathrm{~h}$ of light chopping (biased at $0 \mathrm{~V}_{\mathrm{RHE}}$ ).

\section{Experimental section}

\section{Electrodeposition of cuprous oxide}

The working electrodes were fabricated on Au-coated TEC-15 F:SnO 2 substrates (FTO, NSG glass). FTO-glass was cut into $1 \times 3 \mathrm{~cm}^{2}$ pieces and then cleaned by sonication sequentially in soapy water (15 $\mathrm{min})$, acetone (15 $\mathrm{min})$, ethanol (15 min), and finally in distilled water (15 min). The FTO was coated with $10 \mathrm{~nm} \mathrm{Cr}$, followed by $150 \mathrm{~nm}$ of $\mathrm{Au}$ (by sputtering). The $\mathrm{Cu}_{2} \mathrm{O}$ thin films were electrodeposited from a basic solution of lactate-stabilized copper sulphate $(\mathrm{pH}=12)$ at $30{ }^{\circ} \mathrm{C}$, as described previously. $.^{15} \mathrm{~A} 1 \times 1 \mathrm{~cm}^{2}$ area was left un-deposited to serve as electric contact for experimental characterizations. The deposition conditions were optimized previously and galvanostatic electrodeposition was performed using a two-electrode configuration with Pt mesh as the counter electrode for $105 \mathrm{~min}$ at $-0.1 \mathrm{~mA} \mathrm{~cm}^{-2}$, resulting in a $\mathrm{Cu}_{2} \mathrm{O}$ thickness of roughly $500 \mathrm{~nm}$, as confirmed by the cross sectional SEM - Fig. 1. 


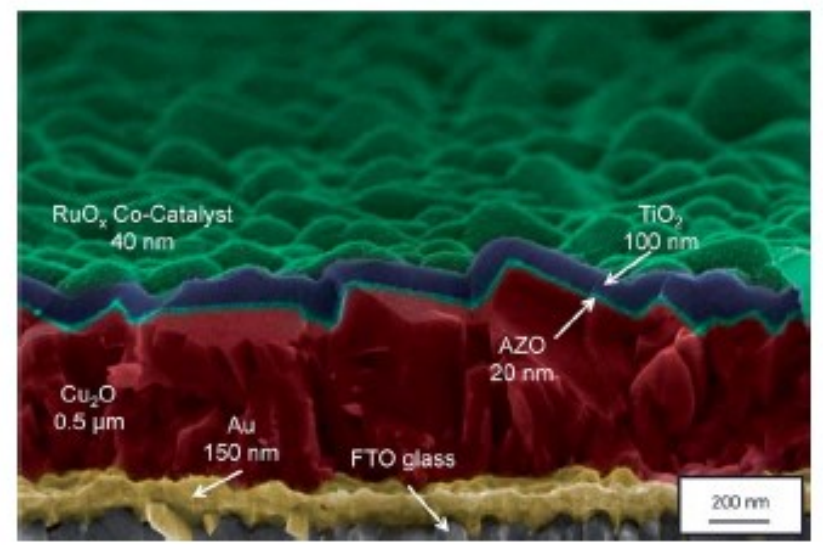

Fig. 1 Cross-sectional SEM image of a photocatode with respective ALD layer thicknesses

\section{Overlayer deposition}

Very thin n-type oxide overlayers were deposited on top of the $\mathrm{Cu}_{2} \mathrm{O}$ using a thermal ALD system (Savannah 100, Cambridge Nanotech). The exposed Au substrate was masked with Kapton ${ }^{\circledR}$ tape and the samples were rinsed with DI water and dried under compressed air prior to deposition. The Al:ZnO (AZO) $(20 \mathrm{~nm})$ and TiO2 $(100 \mathrm{~nm})$ were deposited as described previously. ${ }^{13}$

\section{Steam treatment optimization}

The steam treatment was carried out in a $100 \mathrm{~mL}$ Teflon autoclave. To identify the optimum conditions for the annealing of $\mathrm{Cu}_{2} \mathrm{O}(500 \mathrm{~nm}) / \mathrm{AZO}(20 \mathrm{~nm}) / \mathrm{TiO} 2(100 \mathrm{~nm})$ samples to increase stability, a systematic study of the effect of multiple variables was carried out. The amount of water, treatment duration and annealing temperature were varied and the optimum range of values identified. The samples were observed to be very sensitive to the amount of water in the autoclave, and they would easily dissolve at high temperatures. A small volume ( $200 \mu \mathrm{L})$ of water was added to the autoclave and the sample was sus-pended in the vapor phase to avoid direct contact with water.

In order to identify the range of temperature and duration of steam treatment, a screening design was conducted between $\operatorname{TE}[100,300]^{\circ} \mathrm{C}$ and $\mathrm{t} \mathrm{E}[1,8] \mathrm{h}$. For temperatures above $150^{\circ} \mathrm{C}$ and durations longer than $3 \mathrm{~h}$ the performance of the photo-cathodes was impaired (see examples in Fig. $\mathrm{S}^{\dagger}$ ). For lower temperature and duration, no signifcant change was observed when compared to untreated samples. A careful variation of the temperature $(T)$ and duration (t) intervals with no sample deterioration was conducted with $\mathrm{T}$ between $100^{\circ} \mathrm{C}$ and $150{ }^{\circ} \mathrm{C}$ and $\mathrm{t}$ between $1 \mathrm{~h}$ and $3 \mathrm{~h}$. Table $\mathrm{S} 1+$ shows the parameter values for each experiment. The heating and cooling ramps were fixed at $6{ }^{\circ} \mathrm{C} \mathrm{min}^{-1}$. 
Co-catalyst deposition

For the photoelectrochemical tests, $\mathrm{RuO}_{x}$ and Pt catalysts were used, as described previously. ${ }^{13}$ Briefly, $\mathrm{RuO}_{\mathrm{x}}$ was deposited by galvanostatic photodeposition using $1.3 \mathrm{mM}$ aqueous solution of $\mathrm{KRuO} 4$ and a current density of $28.3 \mu \mathrm{A} \mathrm{cm} \mathrm{cm}^{-2}$ for $15 \mathrm{~min}$ under simulated one sun illumination. Pt was deposited using the same technique with $1 \mathrm{mM}$ solution of $\mathrm{H}_{2} \mathrm{PtCl}_{6}$ and a current density of $-8.5 \mathrm{~mA} \mathrm{~cm}^{-2}$ for $15 \mathrm{~min}$ under simulated one sun illumination.

Thin film characterization

High-resolution scanning electron microscopy (both a FEI XL30 SFEG and also a Zeiss Merlin instrument) with a field emission source operated at $5 \mathrm{kV}$ and a through-the-lens detector for secondary electrons was used to study the morphology of the photocathodes. The crystallinity was evaluated with a Bruker D8 Discover diffractometer, using monochromatic Cu Ka1 radiation $\left(1.540598 \mathrm{~A}^{\circ}\right)$. The Bragg reflections were compared with values from the literature. ${ }^{16}$ Raman scattering measurements were made using a $532 \mathrm{~nm}$ wavelength laser beam and were acquired with a Labram HR800 model of Jobin-Yvon Horiba spectrometer equipped with a microscope for collection of backscattered Raman signals and phonon modes compared with references. 17,18 The thickness and $n, k$ parameters of the ALD titania layer were evaluated using spectroscopic ellipsometry (Sopra GES 5E). The range of photo-energies used in the study was between 1.5 and $5.5 \mathrm{eV}$. The obtained spectra were fitted using the Tauc Lorentz dispersion law (WinELI software) to extract the thickness and $n, k$ parameters of the deposited $\mathrm{TiO}_{2}$ layers. Transmission electron microscopy (TEM) measurements were carried out with a Technai Osiris (FEI, USA) in dark field and bright field modes. An acceleration voltage of $200 \mathrm{kV}$ was applied. A $10 \mu \mathrm{m}$ selected area aperture was used to obtain diffraction patterns of a single layer. The camera length was $330 \mathrm{~mm}$. Cross-section lamellae for TEM were prepared with a Zeiss NVision 40 CrossBeam with focused ion beam (FIB). Diffraction patterns were simulated using JEMS (Version 4.1520, CIME, EPFL, Switzerland).

\section{Photoelectrochemical measurements}

The photoelectrochemical performance of the photocathodes was studied using an Ivium Potentiostat/Galvanostat to acquire the photoresponse under chopped irradiation from a 450 W Xe-lamp (Osram, ozone-free) equipped with an IR/UV - fiter (KG3filter, $3 \mathrm{~mm}$, Schott), calibrated with a silicon diode in order to simulate AM 1.5 illumination $\left(100 \mathrm{~mW} \mathrm{~cm}^{-2}\right)$. A scan rate of $10 \mathrm{mV} \mathrm{s}^{-1}$ in the cathodic direction was used to acquire the current-voltage data.

Measurements were carried out in a three-electrode configuration with the $\mathrm{Cu}_{2} \mathrm{O}$ photocathodes as the working electrode, a Pt wire as the counter-electrode and $\mathrm{Ag} / \mathrm{AgCl} / \mathrm{sat}$. $\mathrm{KCl}$ as the reference electrode, in an electrolyte solution of $0.5 \mathrm{M} \mathrm{Na} 2 \mathrm{SO} 4$ and $0.1 \mathrm{M} \mathrm{KH} 2 \mathrm{PO} 4$ at $\mathrm{pH}$ 5.0. The electrolyte was continuously sparged with nitrogen gas to remove dissolved oxygen. 


\section{Results and discussion}

$\mathrm{TiO} 2$ was proven to be an efficient protective overlayer for $\mathrm{Cu}_{2} \mathrm{O}$ photocathodes in previous studies. ${ }^{11,13,19}$ Nevertheless, the stability of this photocathode is still far from that required for commercial implementation. The biggest limitation in these systems results from electron trapping in the amorphous $\mathrm{TiO} 2$ layer, likely compensated by proton intercalation. We hypothesized that a crystalline overlayer would be more robust, and thus we sought to fabricate a system with a crystalline TiO2 overlayer. It is possible to grow crystalline TiO2 by ALD.20 However, heating the $\mathrm{Cu}_{2} \mathrm{O} / \mathrm{AZO}$ junction to these temperatures (over $200{ }^{\circ} \mathrm{C}$ ) hinders the photoelectrochemical performance significantly..$^{12}$ Post-synthetic heat treatments on a hot plate or in a tubular furnace, in both atmospheric and inert environments led to similar deterioration of the performance. Thus, we have sought a low temperature method to alter the overlayer protective properties without damaging the photovoltaic properties of the semiconductor underlayers.

Hydrothermal treatments have been proven to be useful in the crystallization of oxide films, ${ }^{21}$ including $\mathrm{TiO}_{2},{ }^{22}$ and commonly require temperatures less than $200{ }^{\circ} \mathrm{C}$. Hydro-thermal postsynthetic heat treatments were thus investigated. We found that the oxide materials were sometimes partially or completely dissolved when completely submerged in water in the autoclave at modest temperatures $\left(150^{\circ} \mathrm{C}\right)$. We therefore experimented with steam treatments in which only small amounts of water were added, and care was taken to keep liquid water from contacting the photoelectrode. A systematic study on the temperature and duration of the steam treatment was performed, and the key current-voltage characteristics are presented in Table 1. The plateau photocurrent and the onset potential show no specific trend under steam treatment conditions and the observed variations can be attributed to sample-to-sample variation. On the other hand, stability (here-after evaluated in terms of the time necessary for the photo-current to decrease by $10 \%$ under continuous illumination conditions) reveals a clear relation with steam treatment parameters (Fig. 2). An analysis of variance (ANOVA) was performed and parameters with $p$-values smaller than 0.05 were considered to make significant contribution for the model. The stability showed a correlation with the studied parameters, with determination coefficient $\mathrm{R}^{2}=0.97$. 
Table 1 Observed values of process responses. Stability is evaluated under continuous illumination measurement conditions

\begin{tabular}{lllc}
\hline Run \# & Plateau $\left(\mathrm{mA} \mathrm{cm}^{-2}\right)$ & Onset $\left(\mathrm{V}_{\text {RHE }}\right)$ & $10 \%$ loss $(\mathrm{h})$ \\
\hline 1 & 5.5 & 0.55 & 18 \\
2 & 4.6 & 0.53 & 2 \\
3 & 5.5 & 0.61 & 15 \\
4 & 4.7 & 0.58 & 10 \\
5 & 5.2 & 0.50 & 12 \\
6 & 5.7 & 0.57 & 16 \\
7 & 5.7 & 0.63 & 14 \\
8 & 4.8 & 0.58 & 1 \\
9 & 4.8 & 0.61 & 5 \\
10 & 5.4 & 0.52 & 27
\end{tabular}

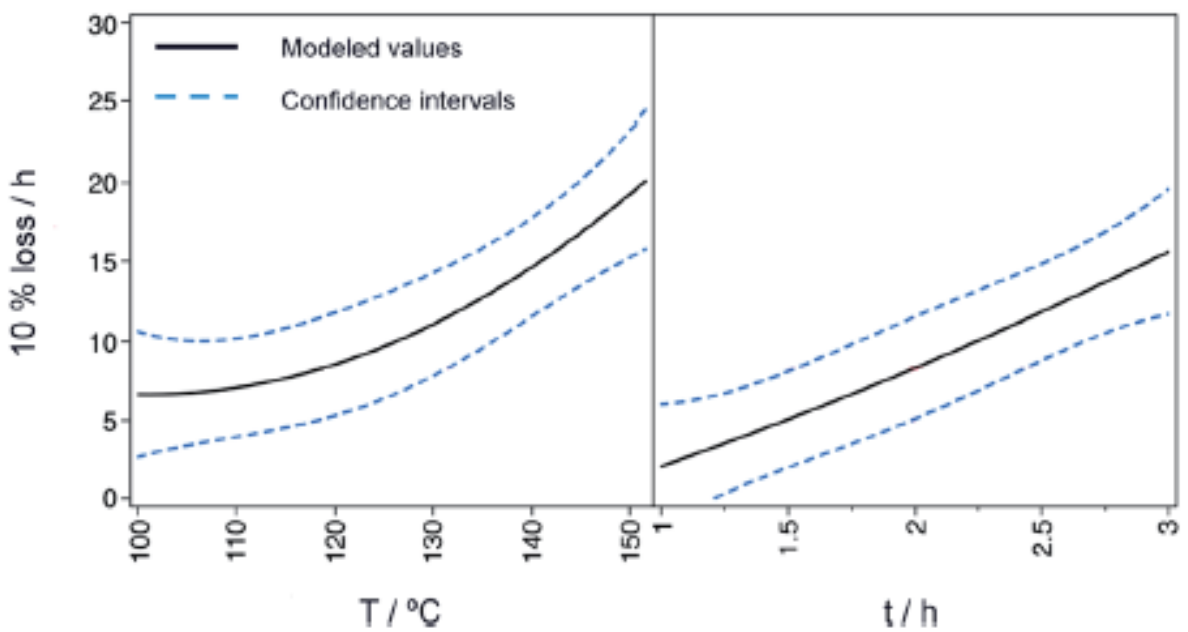

Fig. 2 Stability variation with steam treatment temperature $\left({ }^{\circ} \mathrm{C}\right)$ and duration $(\mathrm{h})$. Stability is evaluated - under continuous illumination measurement conditions - as the time it takes for the initial photo- current to drop to $90 \%$ of its initial value (a $10 \%$ loss). The black lines represent prediction profiles extracted from the statistical analysis on performed measurements and the blue dashed lines represent the corresponding confidence intervals.

Between $100{ }^{\circ} \mathrm{C}$ and $150{ }^{\circ} \mathrm{C}$, a constant increase in stability with temperature and duration of steam treatment is observed (Fig. 2). This is in accordance to what we expected since higher temperatures and annealing times should be related to a higher degree of crystallization. However, at temperatures greater than $150^{\circ} \mathrm{C}$ or duration longer than $3 \mathrm{~h}$ a steep decrease in performance is observed due to the negative impact on the photo-voltaic properties of the 
semiconducting underlayers. We believe that at high temperatures the doping levels of $\mathrm{Cu}_{2} \mathrm{O}$ (which are based on copper vacancies) are altered, thus affecting the performance.

The highest stability was achieved at $150{ }^{\circ} \mathrm{C}$ for $3 \mathrm{~h}$ and the corresponding sample is characterized in Fig. 3 and compared with a photocathode with no steam treatment. The onset is not significantly improved but a plateau photocurrent increase between $2 \%$ and $10 \%$ is observed in the steam treatment case (Fig. 3a). We previously reported the activation of the photo-electrodeposited catalyst after a few linear sweeps, accompanied with a fill factor improvement. ${ }^{13}$ With steam treatment, under measurement conditions, not only does the fill factor increase, with a steep photocurrent increase at $\approx 0.5 \mathrm{~V}_{\mathrm{RHE}}$, but also the onset is shifted anodically by $70 \mathrm{mV}$ (Fig. 3b). The stability is greatly enhanced, recording only $10 \%$ loss over 50 $\mathrm{h}$ biased at $\mathrm{O} \mathrm{V}_{\mathrm{RHE}}$ in the $\mathrm{pH} 5.0$ phosphate-sulfate electrolyte under light chopping (Fig. 3c). This is by far the most stable $\mathrm{Cu}_{2} \mathrm{O}$ photocathode reported to date.
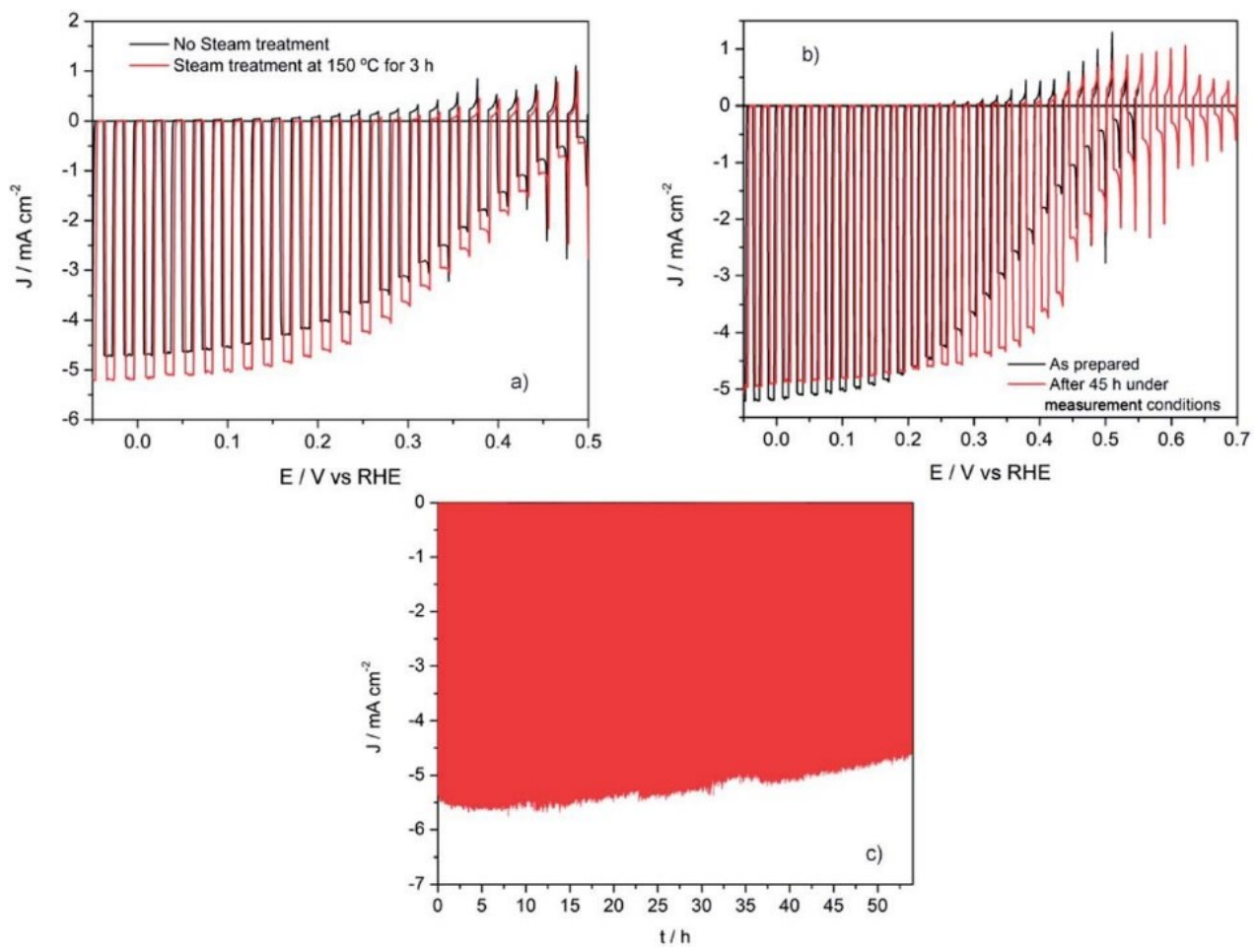

Fig. 3 Current-voltage characteristics of a composite Cu2O photocathode: (a) comparison between the performance of a photocathode as prepared and with steam treatment at 150 ${ }^{\circ} \mathrm{C}$ for $3 \mathrm{~h}$, (b) linear sweep of the photocathode with steam treatment before and after 45 h stability measurement under light chopping, and (c) chronoamperometric stability measurement biased at 0 VRHE. All measurements were carried out in $\mathrm{pH} 5.0$ phosphatesulfate electrolyte under light chopping. 
These results motivated us to study the reason for the improved performance. Park et al. have reported on the crystallization of semiconductor thin films with hydrothermal treatments. ${ }^{21}$ Improved crystallinity in the $\mathrm{TiO} 2$ would explain the superior performance as it is more resistant to electron trapping and/or etching in the slightly acidic medium. XRD and Raman spectra were obtained on photocathodes with different steam treatments to search for crystalline phases (Fig. 4). XRD spectra were unable to identify any $\mathrm{TiO} 2$ crystallization, even for samples that were subjected to extreme conditions, and were quite similar to photocathodes with no steam treatment. ${ }^{12}$ Raman spectroscopy showed very broad bands at 397,518 and $639 \mathrm{~cm}^{-1}$ that could correspond to the anatase phase, but are too faint to suggest crystallinity of the $\mathrm{TiO} 2$ unambiguously. Given the rough nature of the photocathode surface and the coincidental Bragg diffraction peaks of different layers, any crystallization of the $\mathrm{TiO} 2$ protective layer is inconclusive. Therefore, grazing-incidence XRD (GIXRD) was performed on a TiO2-coated quartz substrate $(100 \mathrm{~nm} \mathrm{TiO2})$ to eliminate any contribution from the remaining layers of the photocathode, and revealed that the $\mathrm{TiO} 2$ was amorphous after the steam treatment at $150{ }^{\circ} \mathrm{C}$ for $3 \mathrm{~h}$ (Fig. S3at).
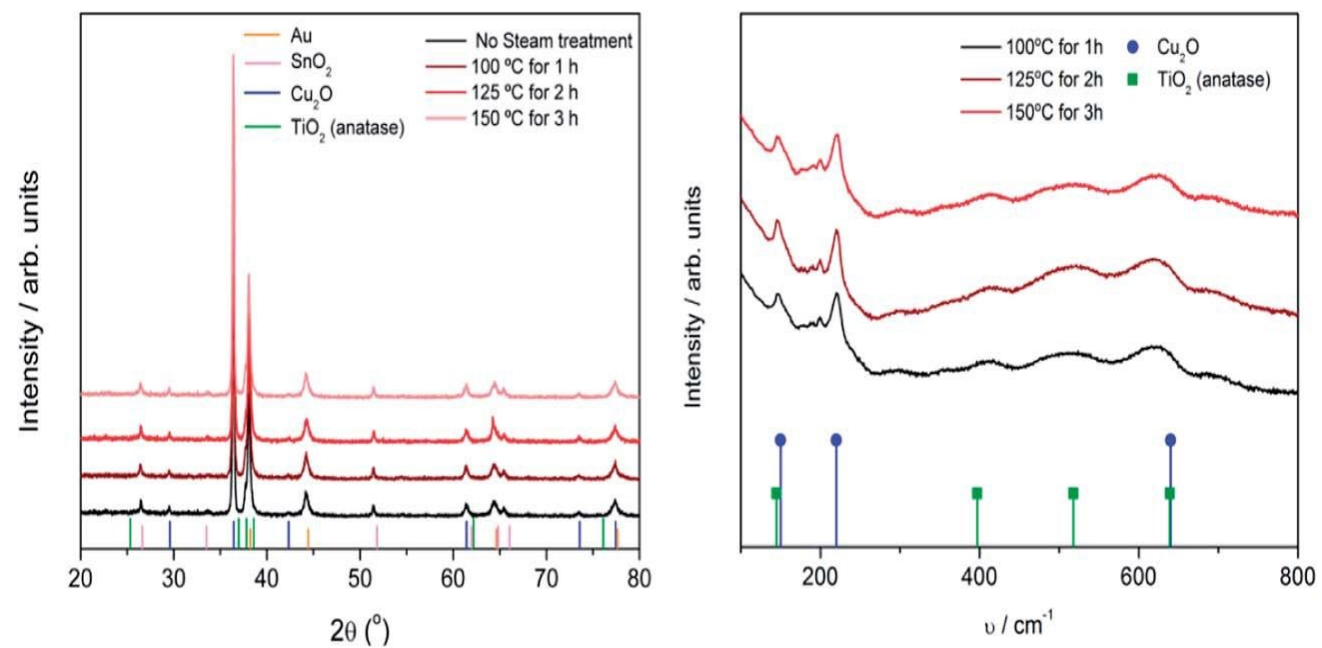

Fig. 4 X-ray diffraction patterns with corresponding layer Bragg reflections (left) and Raman spectra with phonon modes of $\mathrm{Cu}_{2} \mathrm{O}$ and $\mathrm{TiO}_{2}$ of steam treatment samples under different conditions (right).

These two techniques have proven that no major crystalline transition on the $\mathrm{TiO} 2$ is obtained under these steam treatment conditions. Even so, small crystalline domains or surface crystallization (in the order of a few atomic layers) could have formed and not detected by these techniques. Ellipsometry was performed on samples of $100 \mathrm{~nm}$ ALD TiO2 on Si wafer substrates (Fig. $\mathrm{S}_{\mathrm{b}} \mathrm{b}^{+}$) to search for density modifications usually observed in crystallizations. No densification was observed with the steam treatment, expected for an amorphous to crystalline transition. ${ }^{12}$ Since XRD measurements extract a diffraction pattern from all layers, we have chosen transmission electron microscopy (TEM) to selectively investigate the TiO2 layer more precisely. Fig. 5a shows the bright field image of the hydrothermally treated electrode crosssection where the red circle marks the position seen in the dark field (DF) image (Fig. 5b). The DF image was obtained from the selected area diffraction pattern (SADP) shown the bottom of Fig. 5c, and the selected area is shown in the inset of Fig. 5a. The SADP was analyzed using ring patterns simulated in JEMS. This analysis shows the presence of anatase as well as brookite, however, with these few reflections it is difficult to assign the rings accurately. The diffraction 
pattern confirms that the TiO2 layer is largely amorphous and has only a few crystalline domains. These crystalline domains visible in the DF image (Fig. 5b), reveal the presence of crystallinity in our hydrothermally treated $\mathrm{TiO} 2$ overlayer, which could not be resolved in XRD nor in ellipsometric surface density measurements. However, following the same TEM analysis for the reference sample, a similar diffraction pattern was found (inset of Fig. $5 \mathrm{~d}$ ) and crystalline domains are shown to be present as well in the DF image (Fig. $5 \mathrm{~d}$ ). Thus, it could not be concluded that the steam treatment can largely crystallize the ALD grown TiO2 overlayer. Since these crystalline domains have the size of the thickness of the layer it is most probable that they have grown epitaxially during the deposition. Previously, we showed that improvement of the electrical properties of the protective layers can enhance stability by decreasing recombination and electron traps. ${ }^{12,13}$ In TiO2, oxygen vacancies are charge balanced by $\mathrm{Ti}^{3+}$ states that absorb light, and can act as recombination centers. In order to see if the steam treatment changes the defect chemistry of the TiO2 by eliminating oxygen vacancies, we studied the donor density by Mott-Schottky plot (Fig. 6). We can observe, from Table 2, that there is no significant difference in the flatband potential between samples with/without steam treatment. We have noted a slight decrease in the donor density after steam treatment, which is likely due to the filling of oxygen vacancies in the $\mathrm{TiO} 2$ by oxygen in the autoclave during the annealing treatment. However, it is unclear how this relatively modest change could result in dramatic improvements in the stability that we observe in the photocathodes.
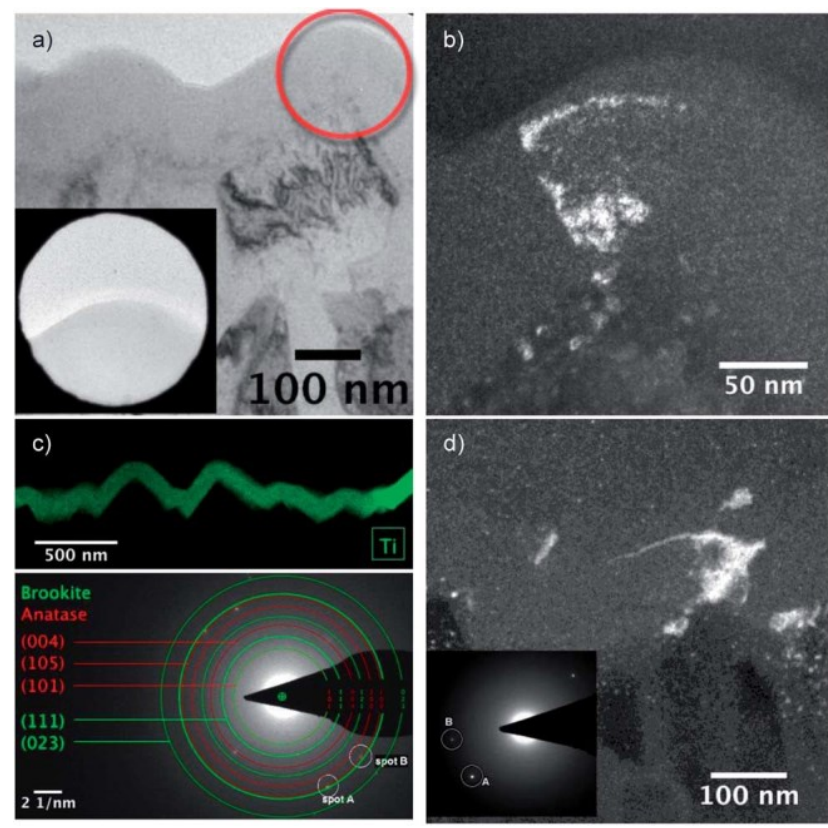

Fig. 5 TEM analysis of the hydrothermally treated sample compared to the reference sample. (a) Bright field image of the hydrothermally treated electrode cross-section (from top to bottom: amorphous carbon/TiO2/AZO/Cu2O). The inset shows the area selected by the aperture for the diffraction pattern shown at the bottom of (c). (b) Dark field image of the position marked in red in (a) and obtained from spot B in the diffraction pattern in (c). Crystalline domains are visible in the $\mathrm{TiO}_{2}$ layer and small crystallites in the $\mathrm{ZnO}$ layer underneath. (c) Top: EDX analysis of the $\mathrm{TiO}_{2}$ layer showing homogenous coverage throughout the film. Bottom: selected area diffraction pattern of $\mathrm{TiO}_{2}$ shown in the inset of (a). Spot B (anatase (105) plane) was used for the dark field image in (b). (d) Dark field image of the reference sample without hydrothermal treatment obtained from spot $A$ in the inset. 
With no clear changes in the crystallinity or the band alignment of the TiO2 overlayer, we decided to take a closer look at the morphology. Under the conditions of the steam treatment (water vapor under pressure), the surface of the $\mathrm{TiO} 2$ will undergo continuous modification due to the repeated cleavage and condensation of Ti-O-Ti bonds in lesser-strained forms (surface relaxation). This bond modification is usually associated with a smoothing of the surface. ${ }^{22-24}$ Fig. 7 shows the surface morphology of the $\mathrm{TiO} 2$ overlayer prior to and after steam treatment. It is clear that a morphological change has occurred. Prior to treatment the $\mathrm{TiO} 2$ reveals a significant roughness with a grainy structure whereas the steam treatment gave a much smoother surface. The thickness profile of the $\mathrm{TiO} 2$ protective layer was also studied by cross-sectional TEM. Different thicknesses of the TiO2 layer were measured along the surface and a clear trend was identified. In agreement with a previous literature report, the thickness of the TiO2 on top of underlayer grain boundaries is larger and at the grain tips is smaller. ${ }^{25}$ Before steam treatment, at the $\mathrm{Cu}_{2} \mathrm{O}$ grain boundaries (represented in red in Fig. $7 \mathrm{c}$ ) the $\mathrm{TiO} 2$ is thicker than at the grain tips with an average difference of $26 \mathrm{~nm}$. After steam treatment the thickness is much more equally distributed with a thickness standard deviation $\sigma=3 \mathrm{~nm}$.

This smoothing process likely patches any defects and cracks in the TiO2 surface as well as improves the quality of the surface for more homogeneous catalyst deposition. In our system, electrodeposition consists of the formation of a layer on a conducting substrate occurring through the electrochemical reduction of higher valence ions dissolved in a suitable aqueous solvent. As electrons flow through the substrate they are transferred to the ions in solution near the surface promoting their reduction and precipitation to form a film. When the substrate is a conducting metal (resistivity on the order of $10^{-9} \Omega \mathrm{m}$ ), electrons flow easily to the surface and are readily available to assist electrodeposition evenly across the surface. On the other hand, when the substrate is several orders of magnitude less conductive, thickness variations on the order of a few nanometers have a large influence on the potential distribution across the surface, resulting in heterogeneous electrodeposition, as the authors verified in previous reports. ${ }^{26-28}$ Since TiO2 is not a conductor $(\rho \approx 1-10 \Omega \mathrm{m}),{ }^{29}$ the rough surface of the amorphous $\mathrm{TiO} 2$ creates an irregular current density distribution across the surface, yielding a granular $\mathrm{RuO}_{\mathrm{x}}$ co-catalyst structure, typical in non-uniform electrodeposition (Fig. S4a†). As the surface turns smooth, the current distribution becomes more regular and the co-catalyst is more homogeneous (Fig. S4bt). Homogeneous deposition of the catalyst on the surface ensures that injected photoelectrons in the $\mathrm{TiO} 2$ will be efficiently extracted, thus minimizing the likelihood of electron trapping in the overlayer. Also, the smoothing and healing of small cracks in the overlayer prevent direct contact of the electrolyte with the $\mathrm{Cu}_{2} \mathrm{O}$ layer, greatly increasing the stability of the system.

The surface morphology of the photocathode is again modified upon deposition of the $\mathrm{RuO}_{x}$ (Fig. 8) offering a higher surface area than the smooth TiO2 layer, which is a clear advantage for catalysis. We have also studied the morphology of the catalyst after a long term stability measurement. After $20 \mathrm{~h}$ under continuous illumination conditions, it appears that the catalyst has undergone a large morphological change, by becoming pasty and cracked (Fig. $8 \mathrm{e}$ and f). It appears that under extended hydrogen evolution conditions, the surface continues to rearrange and smoothen. The cracks that are observed in the SEM images likely occur only after drying in the high vacuum conditions of the SEM.

To support our theory regarding homogeneous electrode-position, we tested a Pt co-catalyst. $\mathrm{Pt}$ is expected to be less stable than RuOx, as previous results indicated, ${ }^{13}$ but we expected that a more homogeneous catalytic layer of Pt on top of the TiO2 would facilitate charge extraction 
and therefore increase stability. A comparison between both catalysts is rep-resented in Fig. 9. We observed an onset potential of $+0.64 \mathrm{~V}_{\mathrm{RHE}}$ and a plateau photocurrent of greater than $5.5 \mathrm{~mA} \mathrm{~cm}^{-2}$ at $0 \mathrm{~V}_{\text {RHE }}$ (Fig. 9a). With a plateau photocurrent $10 \%$ greater than that for $\mathrm{RuO}_{\mathrm{x}}$ and a significant shift in the onset, this represents a major step forward for unbiased photoelectrochemical tandem systems. The stability of both catalysts is presented in Fig. $9 \mathrm{~b}$ and c. As expected, the Pt was less stable than the $\mathrm{RuO}_{x}$ catalyst, yet is vastly superior to previous photocathodes that employed Pt catalyst. With $10 \%$ loss after $20 \mathrm{~h}$ stability measurement biased at $0 \mathrm{~V}_{\mathrm{RHE}}$ in the $\mathrm{pH} 5.0$ phosphate-sulfate electrolyte under light chopping, this level of stability represents an improvement of $2000 \%$ over previous results. ${ }^{12}$

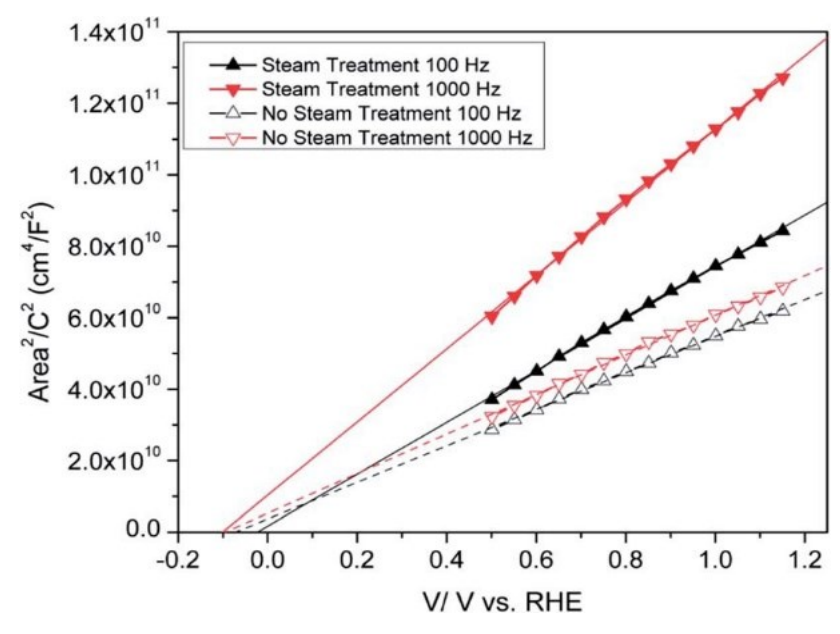

Fig. 6 Mott-Schottky plot of TiO2 films prepared using hydrogen peroxide precursor on FTO, measured in $1 \mathrm{M} \mathrm{NaOH}$ electrolyte. Steam conditions were $150^{\circ} \mathrm{C}$ for $3 \mathrm{~h}$.
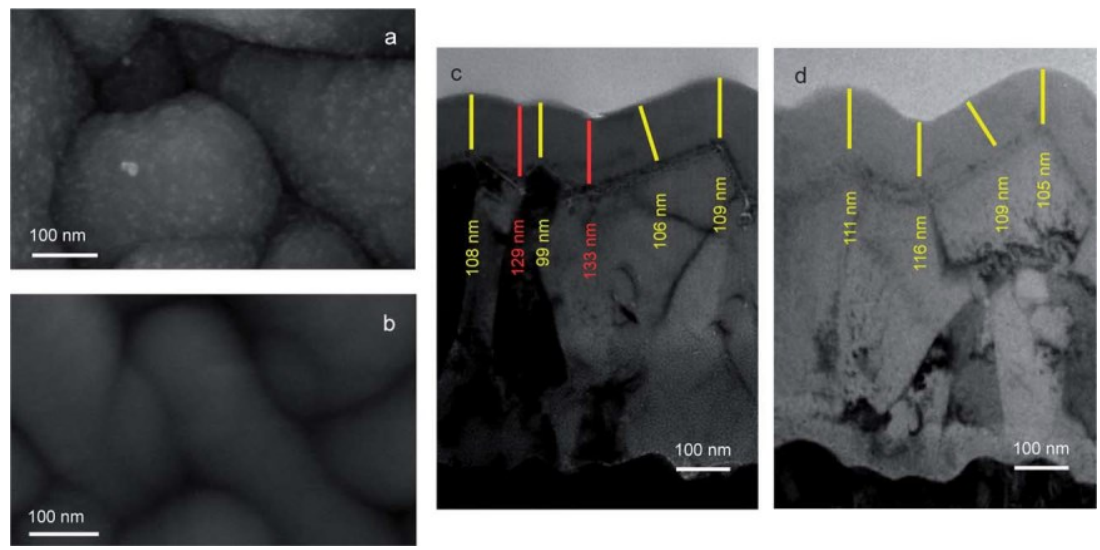

Fig. 7 Surface morphology of the TiO2 overlayer (left) and TEM cross-section (right) of an as prepared sample ( $\mathrm{a}$ and $\mathrm{c}$ ) and a sample with steam treatment at $150^{\circ} \mathrm{C}$ for $3 \mathrm{~h}$ (b and d). It is clear that the surface experienced major morphological changes upon steam treatment. The $\mathrm{TiO}_{2}$ thickness was measured in different places of both samples and the larger thicknesses are marked in red. 

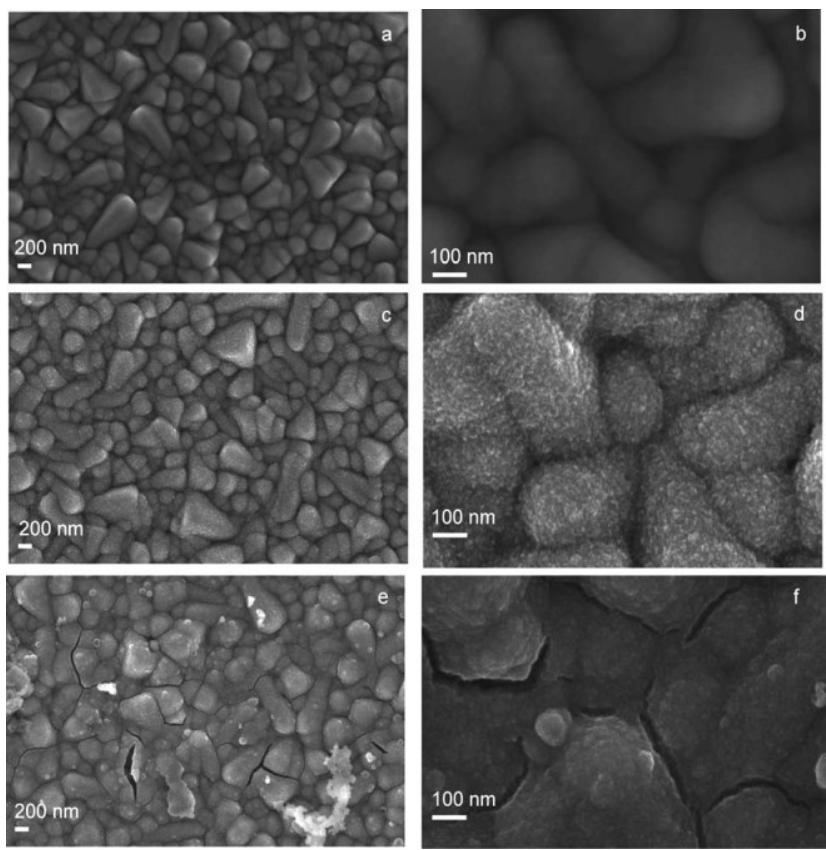

Fig. 8 SEM top view ( $a$ and b) of $\mathrm{TiO}_{2}$ after steam treatment at $150^{\circ} \mathrm{C}$ for $3 \mathrm{~h}$; (c and d) photoassisted electrodeposited $\mathrm{RuO}_{x}$ catalyst; (e and f) active area after $20 \mathrm{~h}$ under illumination conditions.
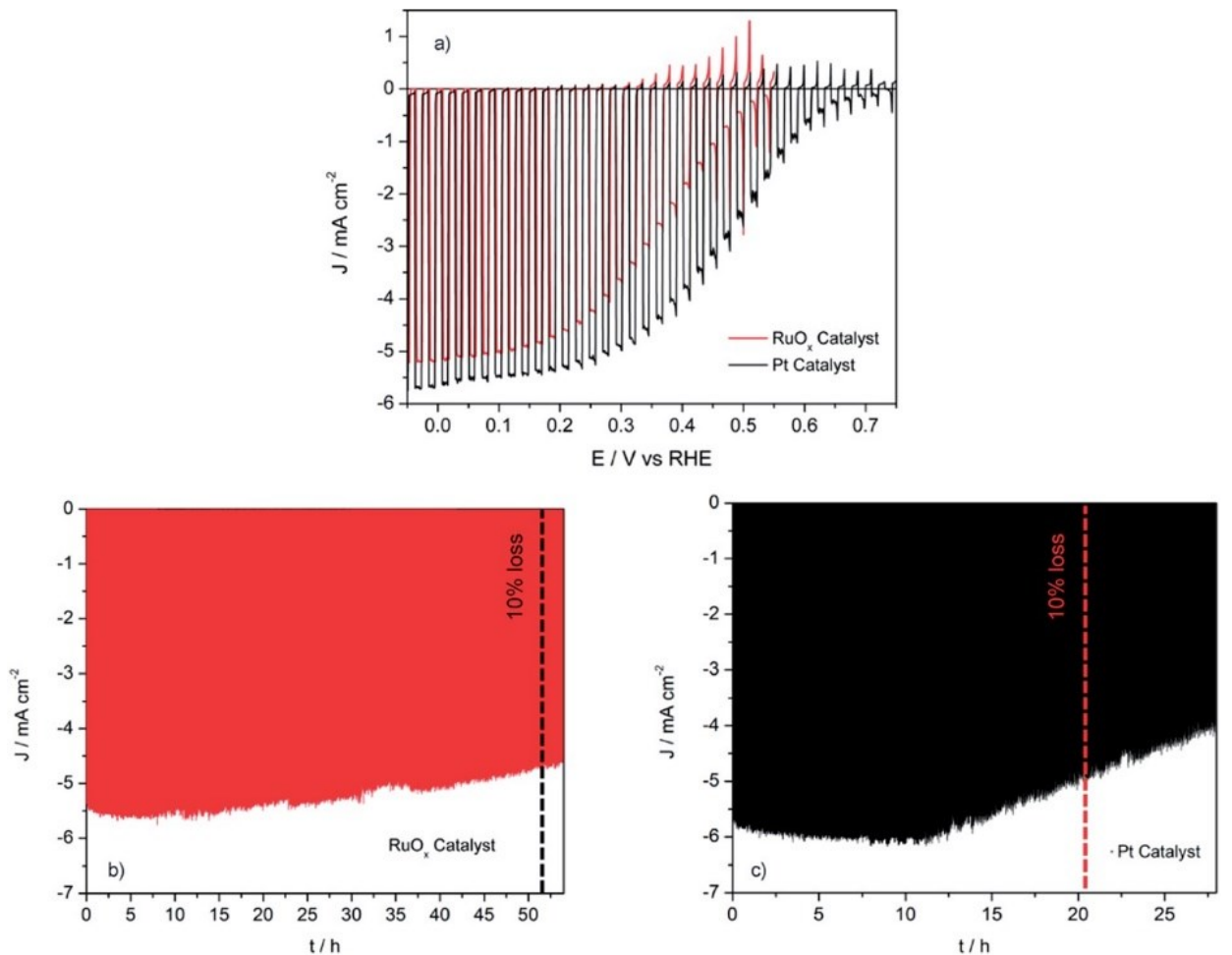

Fig. 9 Current-voltage characteristics of a composite $\mathrm{Cu}_{2} \mathrm{O}$ photocathode after steam treatment at $150^{\circ} \mathrm{C}$ for $3 \mathrm{~h}$ : (a) comparison between the performance of a photocathode with $\mathrm{RuO}_{x}$ and $\mathrm{Pt}$ as catalyst; chronoamperometric stability measurement biased at 0 VRHE in the pH 5.0 phosphate-sulfate electrolyte under light chopping with (b) RuOx and (c) Pt as catalyst. 
Table 2 Comparison of electrical properties of the $\mathrm{TiO}_{2}$ films with/ without steam treatment. Flatband and donor density extracted according to the Mott-Schottky equation $^{19}$

\begin{tabular}{|c|c|c|}
\hline Treatment & $E_{\mathrm{fb}}\left(\mathrm{V}_{\mathrm{RHE}}\right)$ & $N_{\mathrm{D}}\left(\mathrm{cm}^{-3}\right)$ \\
\hline No steam treatment ${ }^{a}$ & -0.10 & $3.5 \times 10^{19}$ \\
\hline Steam treatment for $3 \mathrm{~h}$ at $150^{\circ} \mathrm{C}$ & -0.09 & $2.2 \times 10^{19}$ \\
\hline
\end{tabular}

\section{Conclusions}

A simple low-cost solution was developed to greatly enhance the $\mathrm{Cu}_{2} \mathrm{O}$ photocathode stability. Steam treatments employed on $\mathrm{Cu}_{2} \mathrm{O} / \mathrm{AZO} / \mathrm{TiO} 2$ photocathodes allow for stability improvement using both $\mathrm{RuO}_{x}$ and Pt co-catalysts. Not only did we obtain record breaking stabilities in both cases, we also uncovered the critical importance of morphological changes of the surface in generating these high-stability photocathodes.

\section{Acknowledgements}

J. Azevedo, P. Dias and C. T. Sousa are grateful to the FCT SFRH/BD/79207/2011 PhD grant, SFRH/BD/81016/2011 PhD grant and postdoctoral grant SFRH/BPD/82010/2011, respectively. We also acknowledge the FCT through the project H2Solar (PTDC/EQU-EQU/104217/2008). M. Ste $\cdot \mathrm{k}$ acknowledges startup funds from the University of South Carolina. J. P. Ara'ujo acknowledges NORTE-070124-FEDER-000070 Multifunctional Nanomaterials funded by FEDER and CCDRN. L. Steier, M. Graetzel and S. D. Tilley gratefully acknowledge the Swiss Federal Office for Energy (PECHouse Competence Center, contract number SI/500090-02), the PECDEMO project (co-funded by Europe's Fuel Cell and Hydrogen Joint Undertaking under Grant Agreement no. 621252), and the PHOCS project (European Union, ENERGY 2012-10.2.1, Future Emerging Technologies Collaborative Project no. 309223) for the - nancial support. M. Graetzel thanks the European Research Council for the - nancial support under the Advanced Research Grant (ARG 247404) "Mesolight." Additionally, we thank the Interdisciplinary Centre for Electron Microscopy (CIME) and in particular Dr Duncan Alexander for helping with the HR-TEM analysis.

\section{References}

1. J. Turner, Science, $1999,285,687-689$.

2. A. Fujishima and K. Honda, Nature, 1972, 238, 37-38.

3. P. J. Boddy, J. Electrochem. Soc., 1968, 115, 199-203.

4. K. Sivula, F. Le Formal and M. Gratzel, ChemSusChem, 2011, 4, 432-449.

5. C.-Y. Lin, Y.-H. Lai, D. Mersch and E. Reisner, Chem. Sci., 2012,3,3482.

6. L. Wang, C.-Y. Lee and P. Schmuki,J. Mater. Chem. A, 2012, 1, 212.

7. Tang, W.-J. Yin, M. A. Matin, H. Wang, T. Deutsch, M. M. Al-Jassim, J. A. Turner and Y. Yan, J. Appl. Phys., 2012, 111,073502.

8. M. Stefik, M. Cornuz, N. Mathews, T. Hisatomi, S. Mhaisalkar and M. Gratzel, Nano Lett., 2012, 12, 5431- 5435. 
9. F.F.Abdi, L. Han, A.H.M.Smets, M.Zeman, B.Damand R. van de Krol, Nat. Commun., 2013, 4, 1-18.

10. S. W. Boettcher, E. L. Warren, M. C. Putnam, E. A. Santori, D. Turner-Evans, M. D. Kelzenberg, M. G. Walter, J. R. McKone, B. S. Brunschwig, H. A. Atwater and N.S. Lewis, J. Am. Chem. Soc., 2011, 133, 1216-1219.

11. A. Paracchino, V. Laporte, K. Sivula, M. Gratzel and E. Thimsen, Nat. Mater., 2011, 10,456461.

12. A. Paracchino, N. Mathews, T. Hisatomi, M. Ste国, S. D. Tilleyand M. Gratzel, Energy Environ. Sci., 2012, 5, 8673.

13. S. D. Tilley, M. Schreier, J. Azevedo, M. Ste?lk andM. Graetzel, Adv. Funct. Mater., 2014, 24, 303-311.

14. E. R. Kötz and S. Stucki, J. Appl. Electrochem., 1987, 17, 1190-1197.

15. A. Paracchino, J. C. Brauer, J.-E. Moser, E. Thimsen and M. Graetzel, J. Phys. Chem. C, 2012, 116, 7341-7350.

16. R. T. Downs and M. Hall-Wallace, Am. Mineral., 2003, 88, 247-250.

17. W. F. Zhang, Y. L. He, M. S. Zhang, Z. Yin and Q. Chen, J. Phys. D:Appl. Phys., 2000, 33, 912-916.

18. H. Solache-Carranco, G. Juarez-Diaz, M. Galvan-Arellano, J. Martinez-Juarez, R. Romero-

Paredes and R. Pena-Sierra, presented at $5^{\text {th }}$ International Conference on Electrical Engineering, Computing Science and Automatic Control, 2008, 421-424.

19. G. Morales-Guio, S. D. Tilley, H. Vrubel, M. Gratzel and X. Hu, Nat. Commun., 2014, 5, 3059.

20. M. Reiners, K. Xu, N. Aslam, A. Devi, R. Waser and Hoffmann-Eifert, Chem. Mater., 2013, 25, 2934-2943.

21. Park, B. L. Clark, D. A. Keszler, J.P. Bender, J. F. Wager, A. ReynoldsandG.S. Herman,Science, 2002,297,65-65.

22. D. R. G. Mitchell, G. Triani and Z. Zhang, Thin Solid Films, 2008, 516, 8414-8423.

23. A. I. Kontos, I. M. Arabatzis, D. S. Tsoukleris, A. G. Kontos, M. C. Bernard, D. E. Petrakis and P. Falaras, Catal. Today, 2005, 101,275-281.

24. O. Lupan and T. Pauporté, J. Cryst. Growth, 2010, 312, 2454-2458.

25. K. Murakami, M. Rommel, B. Hudec, A. Rosová, K. Hušeková, E. Dobrockka, R. Rammula, A. Kasikov, J. H. Han, W. Lee, S. J. Song, A. Paskaleva, A. J. Bauer, L. Frey, K. Fröhlich, J. Aarik and C. S. Hwang, ACS Appl. Mater. Interfaces, 2014, 6, 2486-2492.

26. J. Azevedo, C. T. Sousa, J. Ventura, A. Apolinário, M.MendesandJ.P.Araujo,Mater.Res. Express, 2014,1, 015028.

27. J. Azevedo, C. T. Sousa, A. M. Mendes and J. P. Araujo, J. Nanosci. Nanotechnol., 2012,12,91129117.

28. T. Sousa, D. C. Leitao, M. P. Proença, A. Apolinário, J. G. Correia, J.Ventura and J. P. Araujo, Nanotechnology, 2011, 22, 315602-315607.

29. V. Pore, A. Rahtu, M. Leskelä, M. Ritala, T. Sajavaara and J. Keinonen, Chem. Vap. Deposition, $2004,10,143-148$. 